\title{
N-Acyl-Homoserine Lactone Quorum Sensing Switch from Acidogenesis to Solventogenesis during the Fermentation Process in Serratia marcescens MG15
}

\author{
Wensong Jin ${ }^{1,2+}$, Hui Lin $^{1,2+}$, Huifang Gao ${ }^{1,2}$, Zewang Guo ${ }^{1}$, Jiahuan $\mathrm{Li}^{1,2}$, Quanming $\mathrm{Xu}^{1}$, Shujing Sun ${ }^{1,2}$, \\ Kaihui $\mathrm{Hu}^{1,2}$, Jung-Kul Lee ${ }^{3 *}$, and Liaoyuan Zhang ${ }^{1,2 *}$ \\ ${ }^{1}$ College of Life Sciences, Fujian Agriculture and Forestry University, Fuzhou 350002, P.R. China \\ ${ }^{2}$ Gutian Edible Fungi Research Institute, Fujian Agriculture and Forestry University, Gutian 352200, P.R. China \\ ${ }^{3}$ Department of Chemical Engineering, Konkuk University, Seoul 05029, Republic of Korea
}

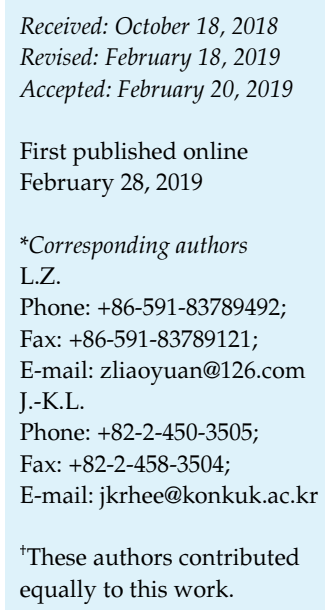

S upplementary data for this paper are available on-line only at http://jmb.or.kr.

pISSN 1017-7825, eISSN 1738-8872 and Biotechnology
$N$-acyl-homoserine lactone quorum sensing (AHL-QS) has been shown to regulate many physiological behaviors in Serratia marcescens MG1. In the current study, the effects of AHL-QS on the biosynthesis of acid and neutral products by S. marcescens MG1 and its isogenic $\Delta s w r I$ with or without supplementing exogenous $N$-hexanoyl-L-homoserine lactone $\left(\mathrm{C}_{6}-\mathrm{HSL}\right)$ were systematically investigated. The results showed that swrI disruption resulted in rapid $\mathrm{pH}$ drops from 7.0 to 4.8 , which could be restored to wild type by supplementing $\mathrm{C}_{6}$-HSL. Furthermore, fermentation product analysis indicated that $\Delta s w r I$ could lead to obvious accumulation for acidogenesis products such as lactic acid and succinic acid, especially excess acetic acid $(2.27 \mathrm{~g} / \mathrm{l})$ produced at the early stage of fermentation, whereas solventogenesis products by $\Delta s w r I$ appeared to noticeably decrease by an approximate $30 \%$ for acetoin during 32-48 $\mathrm{h}$ and by an approximate $20 \%$ for 2,3-butanediol during 24-40 h, when compared to those by wild type. Interestingly, the excess acetic acid produced could be removed in an AHL-QS-independent manner. Subsequently, quantitative real-time PCR was used to determine the mRNA expression levels of genes responsible for acidogenesis and solventogenesis and showed consistent results with those of product synthesis. Finally, by close examination of promoter regions of the analyzed genes, four putative luxI box-like motifs were found upstream of genes encoding acetyl-CoA synthase, lactate dehydrogenase, $\alpha$-acetolactate decarboxylase, and Lys-like regulator. The information from this study provides a novel insight into the roles played by AHL-QS in switching from acidogenesis to solventogenesis in S. marcescens MG1.

Keywords: Serratia marcescens MG1, N-acyl-homoserine lactone quorum sensing, acidogenesis, solventogenesis, switch

\section{Introduction}

Quorum sensing (QS) is a communication system that allows bacteria to monitor their population density through a process of chemical cell-to-cell signaling mediated by diffusible signal molecules [1-3]. Previous studies showed that a considerable number of bacteria employ a QS system to regulate their physiological behaviors, including bioluminescence, biofilm development, virulence factor secretion, and other cellular processes $[4,5]$. Serratia liquefaciens MG1 was isolated from a softened cucumber [6] and was later renamed Serratia marcescens MG1 in 2005 [7]. S. marcescens MG1 can secrete a variety of hydrolytic enzymes with potential applications, including nuclease, lipase, protease, and phospholipase [6, 8]. In addition, S. marcescens MG1 also produces the biosurfactant serrawettin 
[9], which exhibits antimicrobial, antitumor and plant protecting properties. These hydrolytic enzymes and serrawettin production have been shown to be controlled by QS. The $N$-acyl-homoserine lactone (AHL) QS system in S. marcescens MG1 consists of swrI and swrR genes, which belong to the luxIR type of QS system. swrI, a homolog of luxI, is responsible for directing the synthesis of two AHL signal molecules, $\mathrm{N}$-butanoyl-L-homoserine lactone $\left(\mathrm{C}_{4^{-}}\right.$ HSL) and $N$-hexanoyl-L-homoserine lactone $\left(\mathrm{C}_{6}-\mathrm{HSL}\right)$; swrR, a homolog of $l u x R$, is a transcriptional factor that senses and interacts with $\mathrm{C}_{4}$-HSL and $\mathrm{C}_{6}$-HSL autoinducers in the cytoplasm and then binds to its target gene promoters, functioning as either a transcriptional activator or repressor.

Another important property of $S$. marcescens MG1 is that it is capable of producing a large number of neutral compounds (instead of mixed acids), such as acetoin and 2,3-butanediol (two important biobased bulk chemicals [10, 11]), when cultured under fermentation conditions using carbohydrates as a carbon source. To date, the physiological function of 2,3-butanediol has not been clarified. In many cases, Enterobacteriaceae members utilize sugar to produce organic acids, mainly including acetate, lactate, succinate, and formate, resulting in excessive acidification and growth inhibition during the culture process. However, some microorganisms, such as Klebsiella sp., Clostridium acetobutylicum, and Serratia sp., seem to possess the capability of switching their metabolism to the production of neutral products such as acetone, butanol, alcohols, 1,3propanediol, or 2,3-butanediol [12-15]. It was reported that in Aerobacter aerogenes, acetate, among all of the acid and neutral products tested, was found to be the most effective inducer for $\alpha$-acetolactate synthase, $\alpha$-acetolactate decarboxylase, and butanediol dehydrogenase activities, all of which are involved in the conversion of pyruvate to 2,3-butanediol [16]. In S. marcescens MG1, acetate at $40 \mathrm{mM}$ dramatically promotes the mRNA expression levels of Lyslike regulator (slaR), $\alpha$-acetolactate decarboxylase (slaA), and $\alpha$-acetolactate synthase (slaB), but not butanediol dehydrogenase (slaC) [14]. Moreover, fermentation media supplemented with various amounts of sodium acetate increased the productivity of acetoin, and acetate boosted the $\alpha$-acetolactate synthase gene expression in the tested Serratia sp. [17]. Therefore, Serratia sp. and perhaps those microorganisms that have developed defensive strategies against excessive acidification in the culture medium might employ acetate to switch the fermentation process from acidogenesis to solventogenesis.

Interestingly, acetoin and 2,3-butanediol production regulated by the AHL-QS system were uncovered by Rob et al. in the S. plymuthica RVH1 and S. marcescens MG1 strains [18]. Inactivation of splI (a homolog of luxI) in the AHL-QS system showed a sharp decrease in $\mathrm{pH}$ and less acetoin and 2,3-butanediol production, which could be restored by supplementation with exogenous 3-oxo- $\mathrm{C}_{6}$ HSL [18]. Moreover, splR (a homolog of luxR) was found to be a negative regulator of acetoin production [18]. In addition, swrR negatively regulated slaR mRNA expression in S. marcescens MG1; however, $\Delta s w r R$ produced a

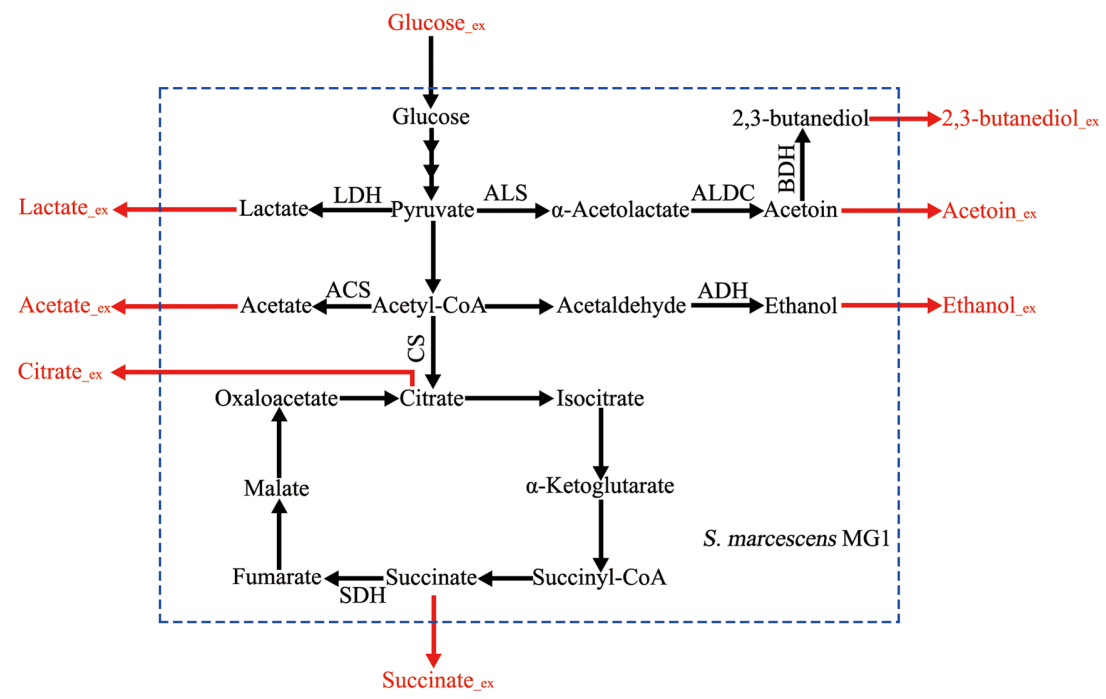

Fig. 1. Fermentation pathways in S. marcescens MG1.

Abbreviations: LDH, lactate dehydrogenase; ACS, acetyl-CoA synthase; CS, citrate synthase; SDH, succinate dehydrogenase; ALS, $\alpha$-acetolactate synthase; ALDC, $\alpha$-acetolactate decarboxylase; $\mathrm{BDH}$, 2,3-butanediol dehydrogenase; $\mathrm{ADH}$, alcohol dehydrogenase. 
comparable amount of 2,3-butanediol as the wild-type strain did [14]. These findings clearly demonstrated that the AHL-QS system is associated with the mixed-acid fermentation pathway and the 2,3-butanediol synthesis pathway. However, how the AHL-QS system in S. marcescens MG1 exerts effects on these pathways remains to be elucidated. A previous finding indicated that regulation of a total of 28 genes was dependent on the $\mathrm{C}_{4}$-HSL-mediated QS system [19], but only $\operatorname{swr} A, \operatorname{lip} B, \operatorname{bsm} A$, and $\operatorname{bamB}$ gene functions were uncovered, the gene products of which are responsible for swarming motility, protein transport and biofilm development in $S$. marcescens MG1, respectively $[9,20,21]$. Functions of the remaining 24 genes remain unknown. In other words, these genes might be involved in biological processes other than those mentioned above. In light of AHL-QS associated with both the $\mathrm{pH}$ value change and the 2,3-butanediol synthesis pathway during S. marcescens MG1 fermentation, we speculated that it might be involved in switching from acidogenesis to solventogenesis. Therefore, we systematically determined the main acid product and neutral product contents generated by $S$. marcescens MG1 during the fermentation process (Fig. 1). Moreover, by applying a quantitative realtime PCR approach, the mRNA expression levels of genes responsible for producing corresponding acids and solvents were analyzed.

\section{Materials and Methods}

\section{Strains, Plasmids, and Culture Conditions}

The strains and plasmids used in this study are listed in Table 1. All strains were routinely maintained in Luria-Bertani (LB) media at $30^{\circ} \mathrm{C}$ for S. marcescens MG1, $\Delta$ swrI, and Chromobacterium violaceum CV026, at $37^{\circ} \mathrm{C}$ for E. coli DH5 $\alpha$ and E. coli S17-1 $\lambda$ pir. Antibiotics were added in the following amounts: $50 \mu \mathrm{g} / \mathrm{ml}$ kanamycin for E. coli S17-1 $\lambda$ pir, $20 \mu \mathrm{g} / \mathrm{ml}$ kanamycin for Chromobacterium violaceum CV026, and $100 \mu \mathrm{g} / \mathrm{ml}$ kanamycin for $\Delta$ swrI. For flask fermentation experiments, seed culture was prepared in LB media. After overnight cultivation, 5\% seed culture of $S$. marcescens MG1 and $\Delta s w r I$ were inoculated into the fermentation medium $(50 \mathrm{ml}$ in a $250 \mathrm{ml}$ shake flask). Fermentation medium for S. marcescens MG1 and $\Delta$ swrI consisted of $7 \%$ glucose, $1.5 \%$ yeast extract, $0.1 \%$ $\mathrm{NH}_{4} \mathrm{H}_{2} \mathrm{PO}_{4}, 0.03 \% \mathrm{MgSO}_{4}$, and $0.01 \% \mathrm{MnSO}_{4}, \mathrm{pH} 7.0$. For the rescue experiments, in parallel with the wild-type strain fermentation, a final concentration of $10 \mu \mathrm{M} \mathrm{C}_{6}$-HSL (Sigma, USA) was added to the $\Delta s w r I$ medium at the initial stage of fermentation. Samples were collected at $8 \mathrm{~h}$ intervals and were used for the analysis of the culture $\mathrm{pH}$ values, dry cell weight, residual sugar, acid products, neutral products, and target gene mRNA expression levels. All flask experiments were performed in triplicate.

\section{Construction of the $\Delta s w r I$ Mutant Strain}

swrI-KO-S and swrI-KO-AS primers were designed according to the released swrI gene sequence in GenBank (GenBank Accession No. AY168877.1) and were subsequently used to amplify partial swrI fragments using the $S$. marcescens MG1 genome as a PCR template. All primers used in this study are provided in Table S1. The amplified DNA fragment was digested with KpnI and ScaI, purified, and ligated to the suicide vector pUTkm1, which was treated with the same enzymes as the swrI fragment. Thus, the marker-exchange plasmid pUT-swrI vector was created. The pUTswrI vector insertion was verified by double enzyme digestion and commercial DNA sequencing. Then, the pUT-swrI vector was transformed into E. coli S17-1 $\lambda$ pir for mating with S. marcescens MG1. Transconjugants were screened on LB agar plates supplemented with $100 \mu \mathrm{g} / \mathrm{ml}$ ampicillin and $100 \mu \mathrm{g} / \mathrm{ml}$ kanamycin. A PCRbased approach was used to confirm disruption of the swrI locus with KR-S and KR-AS primers, which were designed to complement the $\mathrm{Km}^{\mathrm{R}}$ cassette and the endogenous swrI locus outside of the insert in pUT-swrI (data not shown).

\section{Analytic Methods}

The biomass and glucose concentrations in the collected samples were determined by the methods described previously in our laboratory [22]. Gas chromatography (Agilent 7890A, Waldblum, Germany) was used to analyze and quantify ethanol, acetoin, and 2,3-butanediol as described previously [22]. The method for acid product analysis was described as follows: Samples were centrifuged at $10,000 \mathrm{~g}$ for $10 \mathrm{~min}$ to remove strain pellets, and then, acidic ethanol $(80 \mathrm{ml}$ ethanol plus $20 \mathrm{ml}$

Table 1. Bacterial strains and plasmids used in this study.

\begin{tabular}{|c|c|c|}
\hline Strains or plasmids & Characteristics & Reference or source \\
\hline S. marcescens MG1 & Wild Type, $\operatorname{Tc}^{r} A p^{r}$ & CICC 25567 and laboratory stock \\
\hline$\Delta s w r I$ & S. marcescens MG1 harboring swrI deletion mutant, $\mathrm{Tc}^{\mathrm{R}} A \mathrm{p}^{\mathrm{R}} \mathrm{Km}^{\mathrm{R}}$ & This study \\
\hline E.coli S17-1 $\lambda$ pir & recA pro hsdR RP4-2-Tc::Mu-Km::Tn7 & Laboratory stock and [32] \\
\hline C. violaceum $\mathrm{CV} 026$ & $\begin{array}{l}\text { a mutant strain deficient in production of autoinducers } \\
\text { used as quorum sensing reporter organism }\end{array}$ & Laboratory stock and [33] \\
\hline pUT-swrI & pUTKm1 harboring swrI fragment, $\mathrm{Km}^{\mathrm{R}}$ & This study \\
\hline
\end{tabular}


deionized water, adjusted to $\mathrm{pH} 3.0$ by $\mathrm{H}_{3} \mathrm{PO}_{4}$ ) was added to the supernatant, mixed, and then centrifuged again to remove any residual proteins. The obtained supernatant was filtered through a $0.22 \mu \mathrm{m}$ filter membrane (Millipore, Germany). Finally, the lactic acid, acetic acid, citric acid, and succinic acid contents were determined by a high-performance liquid chromatography system (Agilent 1100, Germany) equipped with a Diode Array Detector (DAD). Gradient elution was conducted using a mobile phase starting with $95 \% 0.01 \mathrm{M} \mathrm{KH}_{2} \mathrm{PO}_{4}(\mathrm{pH} 2.05)$ and $5 \%$ organic $\left(100 \%\right.$ methanol) solvent on an SB-Aq C18 column at $30^{\circ} \mathrm{C}$. The flow rate was set at $0.6 \mathrm{ml} / \mathrm{min}$, with a total run time of $30 \mathrm{~min}$. The signal was monitored at $210 \mathrm{~nm}$. Data were collected and calculated according to the strand curve generated by the corresponding standard samples.

\section{Quantitative Real-Time PCR Analysis}

Total RNA from the samples collected was extracted using Trizol reagent (Invitrogen, USA) individually, according to the manufacturer's protocols. cDNA was synthesized from the extracted total RNA with a PrimeScript RT Reagent Kit with gDNA Eraser (Takara, China). All real-time primers with the exception of slaC were designed with Beacon Designer 7 software, based on the available $S$. marcescens WW4 genomic sequence data. slaC real-time primers were used as designed by Rao et al. [14]. Relevant gene expression profiles were determined by StepOne Real-Time PCR (Applied Biosystems, Singapore) with TB Green Premix Ex Taq (TIi RNase H Plus) (Takara). The relative target gene RNA expression levels were analyzed. Data were normalized to 16S RNA expression levels and calculated as $2^{-\Delta \Lambda C T}$. Experiments were performed in triplicate.

\section{Statistical Analysis}

Statistical analyses were performed with GraphPad Prism 7 (GraphPad Software, Inc., USA). The results were expressed as the mean values with SDs and were conducted with two-way ANOVA. A $p$-value of $<0.05$ was considered to be significant.

\section{Results and Discussion}

Effect of swrI Disruption on the Biomass Concentration, pH Profile, and Sugar Consumption of S. marcescens MG1

To test whether $N$-acyl-homoserine lactone quorum sensing (AHL-QS) functions in switching from acidogenesis
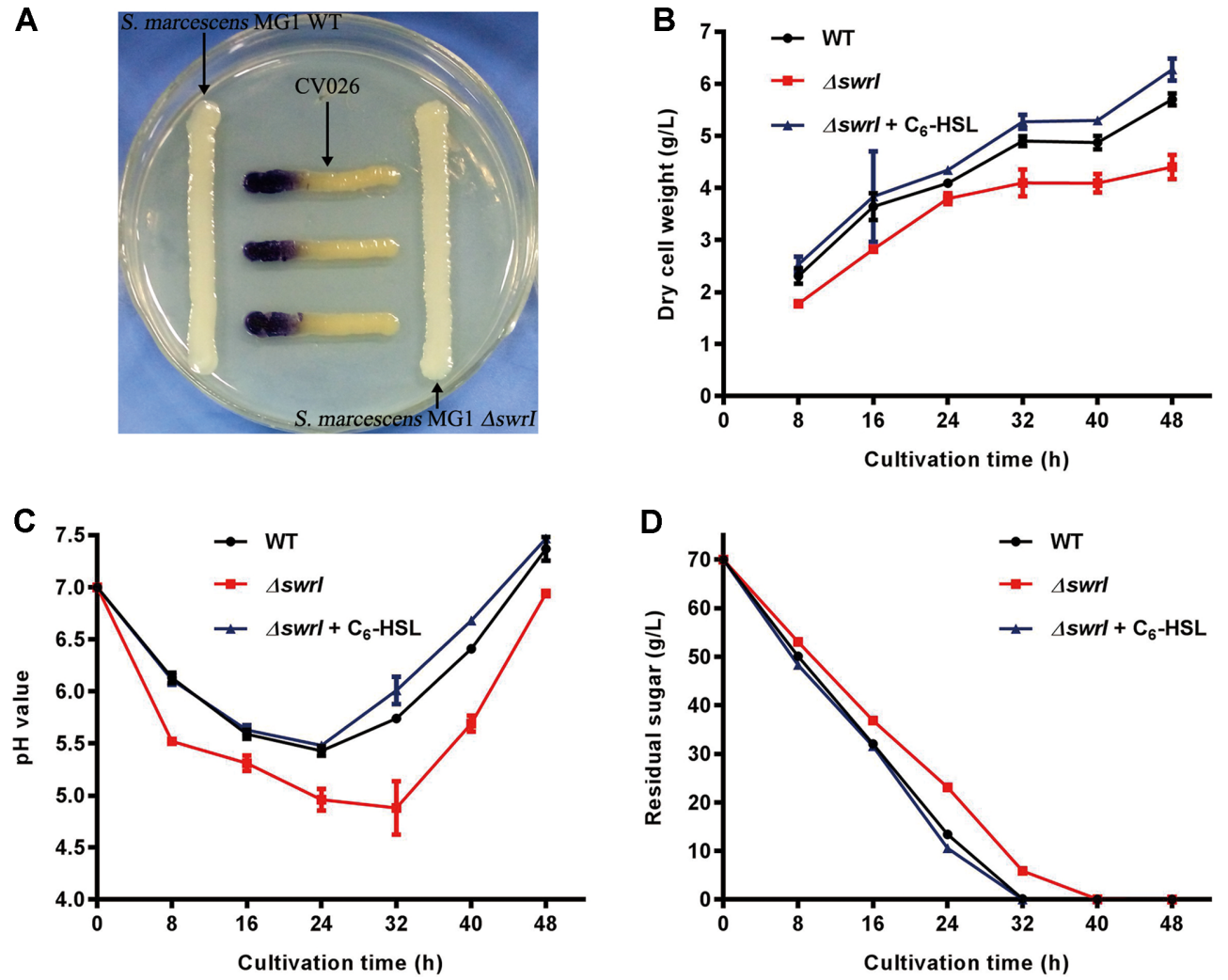

Fig. 2. Effects of swrI disruption on biomass concentration, $\mathrm{pH}$ profile, and sugar consumption of S. marcescens MG1.

Construction of S. marcescens MG1 swrI disruption mutant (A). Samples were collected at the indicated fermentation time points and biomass concentration (B), $\mathrm{pH}$ profile (C), and sugar consumption (D) determination assays for S. marcescens MG1 WT and $\Delta$ swrI complemented with or without $\mathrm{C}_{6}$-HSL were performed. Values are means, and bars indicate standard deviations (SDs) $(n=3)$. 
to solventogenesis, we first used a marker-exchange strategy to construct a swrI disruption mutant. As shown in Fig. 2A, the wild-type strain (WT) efficiently elicited the indicative strain C. violaceum CV026 to produce violacein, which could not be induced by $\Delta s w r I$ under the same tested conditions. This demonstrates that $\Delta s w r I$ lost the capability to synthesize AHL autoinducers and indicates that the endogenous swrI locus was destroyed completely in this mutant strain. Subsequently, disruption of the swrI locus was confirmed by PCR-based analysis (data not shown). The cell growth profile, culture $\mathrm{pH}$, and sugar consumption of WT and $\Delta s w r I$ were evaluated and compared. The cell growth profiles determined by dry cell weight assays showed that WT produced more biomass than $\Delta s w r I$, but slightly less than $\Delta s w r I$ complemented with exogenous $\mathrm{C}_{6}$ HSL, during the whole fermentation process (Fig. 2B). The data presented in Fig. 2B demonstrate that the AHL-QS system positively contributes to the growth rate of S. marcescens MG1 to a certain degree, but it is dispensable for its growth. Our finding is consistent with results discovered by Rob et al. [18] but is in contrast to the data found from Aeromonas hydrophila $\mathrm{AH}-1 \mathrm{~N}$ and $V$. fischeri [23, 24], both of which showed that AHL-QS and AinS (specifically for synthesizing autoinducer-2) QS systems are essential for their growth. In agreement with the strain growth profiles, $\Delta s w r I$ exhibited the lowest sugar consumption kinetics, but the defective phenotype of $\Delta s w r I$ in sugar consumption could be rescued by the addition of exogenous $\mathrm{C}_{6}$-HSL (Fig. 2D). After analyzing the data generated by examining the culture $\mathrm{pH}$ values at different fermentation time points, the corresponding results showed that the $\mathrm{pH}$ values recorded for WT and $\Delta$ swrI supplemented with or without exogenous $\mathrm{C}_{6}$ - $\mathrm{HSL}$ represented inversely typical parabolic curves that reached the baseline at $24 \mathrm{~h}$ for WT and $\Delta s w r I$ supplemented with exogenous $\mathrm{C}_{6}-\mathrm{HSL}$ and at $32 \mathrm{~h}$ for $\Delta$ swrI (Fig. 2C). The lowest $\mathrm{pH}$ value for WT was approximately 5.5 , while that for $\Delta$ swrI was 4.8 during the whole fermentation process. As culture acidifiers
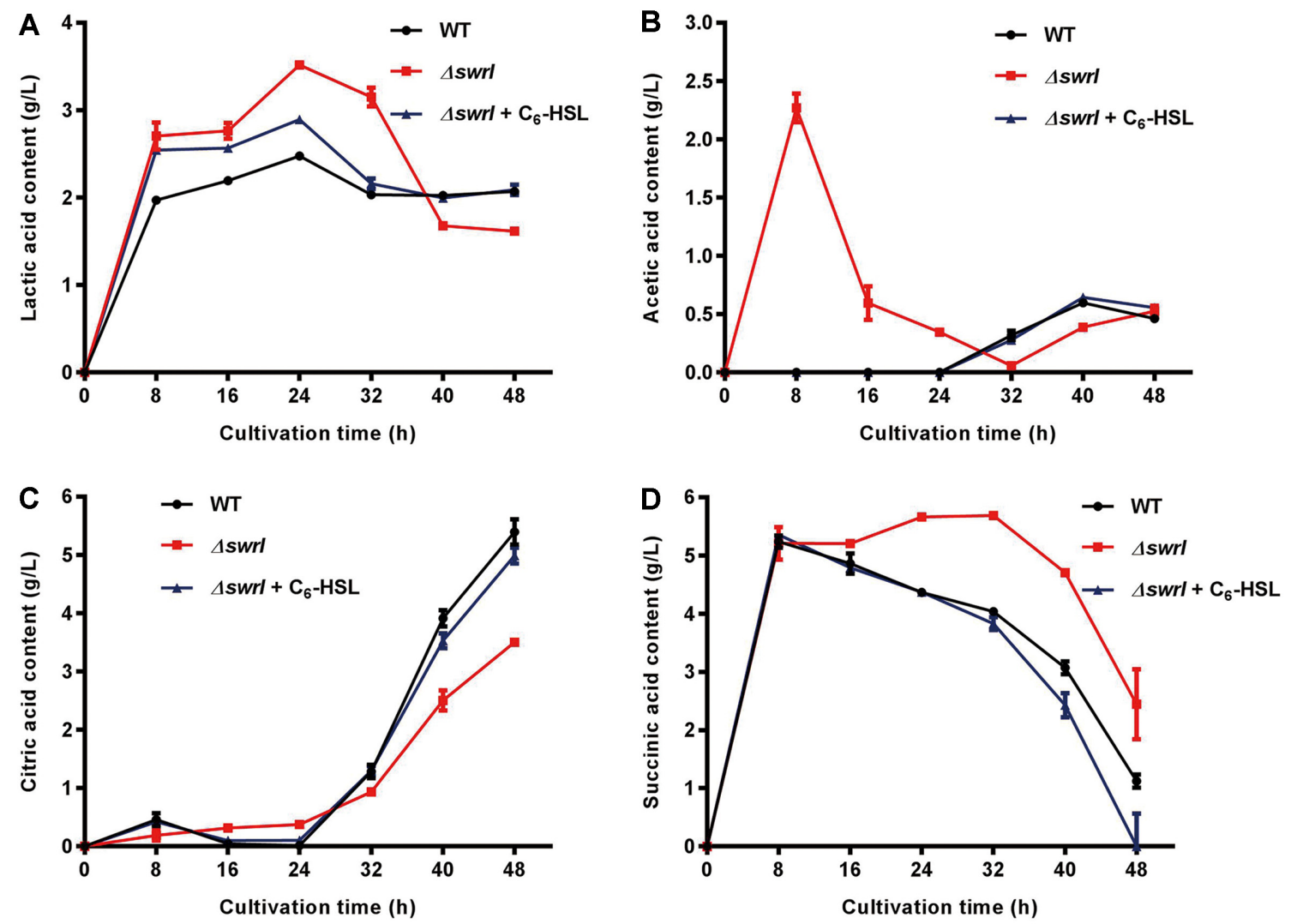

Fig. 3. Effect of swrI disruption on acidogenesis in S. marcescens MG1.

Samples were collected at the indicated fermentation time points and lactic acid content (A), acetic acid content (B), citric acid content (C), and succinic acid content (D) produced by S. marcescens MG1 WT and $\Delta$ swrI complemented with or without $\mathrm{C}_{6}$-HSL were determined. Values are means, and bars indicate standard deviations (SDs) $(n=3)$. 
gradually accumulate, the growth of the fermentation strain gradually ceases [13]. In the case of $S$. marcescens MG1, the higher amount of culture acidifiers accumulated in the spent medium might account for the lower biomass.

\section{Effect of swrI Disruption on Acidogenesis in S. marcescens MG1}

The $\mathrm{pH}$ values of $\Delta s w r I$ culture were dramatically lower compared with those of WT culture during the fermentation process (Fig. 2C) $(p<0.05$, data not shown). To explore the possible factor(s) contributing to this phenomenon, we systematically analyzed the contents of the mixed acid products (Fig. 1), including lactic acid, acetic acid, citric acid and succinic acid, which are the main $S$. marcescens MG1 fermentation acid products secreted to the medium, according to our preliminary experiment results (data not shown). As the fermentation began, WT quickly produced lactic acid and succinic acid. Subsequently, lactic acid contents were maintained at an approximate $2 \mathrm{~g} / \mathrm{l}$ during the whole fermentation process (Fig. 3A), while succinic acid contents gradually decreased after $8 \mathrm{~h}$ fermenting (Fig. 3D), implying that succinic acid must be reused in some way. In contrast, WT produced a slight amount of citric acid and did not produce acetic acid at all during the fermentation period of $0-24 \mathrm{~h}$. After that period, these two acids were concomitantly accumulated. The kinetics of lactic acid, citric acid and succinic acid produced by $\Delta s w r I$ exhibited a similar pattern to those of WT or $\Delta$ swrI complemented with exogenous $\mathrm{C}_{6}$-HSL (Figs. 3A, 3C, and 3D). However, $\Delta s w r I$ produced large amounts of acetic acid as early as the fermentation period of $0-8 \mathrm{~h}$ which was then gradually removed from the media (Fig. 3B). It was reported that $V$. fischeri cells initially excrete acetic acid, and the excess acetic acid can be removed from the media in an AHL-QS-dependent manner [24]. However, our data clearly demonstrate that $\Delta$ swrI can remove excess acetic acid in the medium with an unknown strategy separate from the AHL-QS system, although it was also obvious that AHL-QS is involved in the process of acetic acid production (Fig. 3B). According to the conclusion addressed by Sarah et al., adding the same amount of exogenous acetic acid as generated by the AinS strain to uninoculated medium is sufficient to account for the $\mathrm{pH}$ drop [24]. The large amount of acetic acid produced by $\Delta$ swrI during the fermentation period of 0-24 $\mathrm{h}$ might account for the sharp $\mathrm{pH}$ drop (Figs. 2C and 3B). Since WT generated more citric acid than $\triangle$ swrI (Fig. 3C), the combined contribution of the other three acids analyzed in the culture to acidification
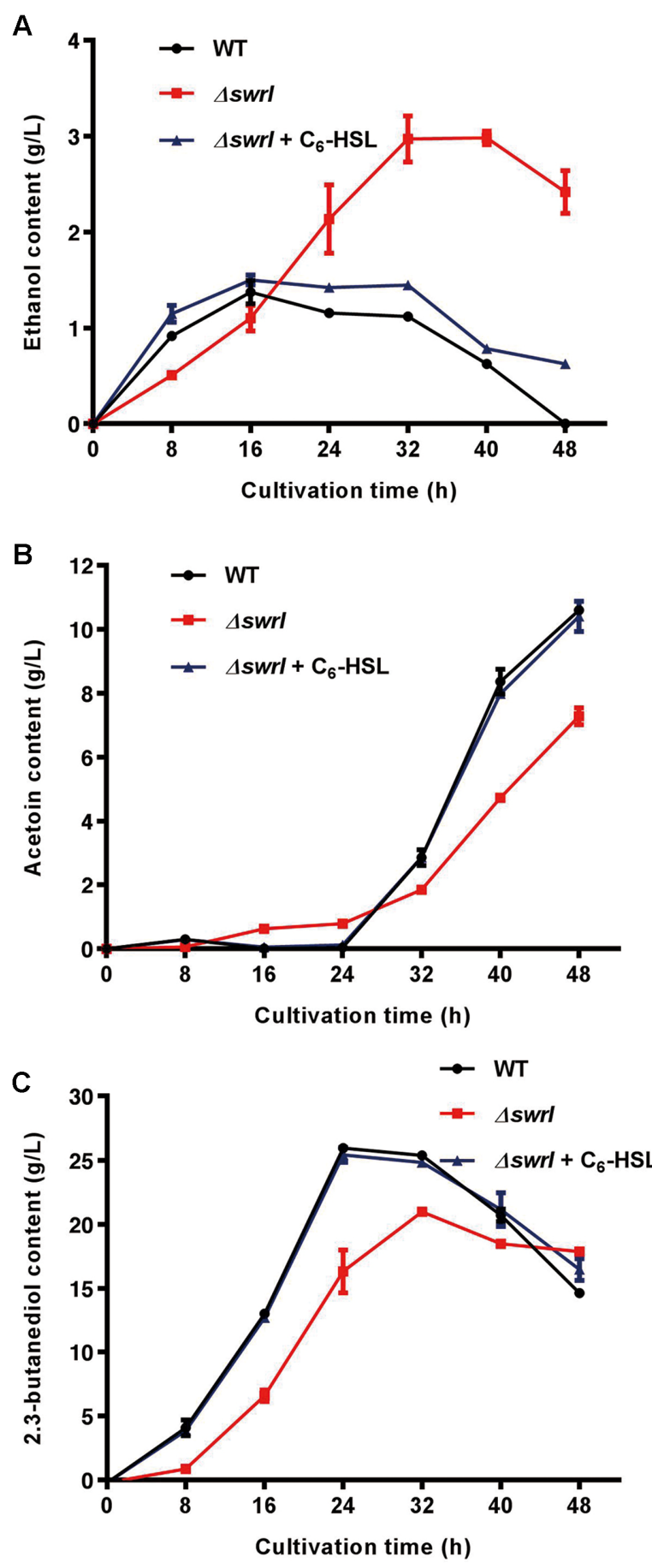

Fig. 4. Effect of swrI disruption on solventogenesis in S. marcescens MG1.

Samples were collected at the indicated fermentation time points. Ethanol content (A), acetoin content (B), and 2,3-butanediol content (C) produced by S. marcescens WT and $\Delta$ swrI complemented with or without $\mathrm{C}_{6}$-HSL were determined. Values are means, and bars indicate standard deviations (SDs) $(n=3)$. 
possibly leads to the phenomenon whereby the $\mathrm{pH}$ values of $\Delta s w r I$ culture were accordingly lower than those of WT culture during the whole fermentation process (Figs. 3 and $2 \mathrm{C})$.

\section{Effect of swrI Disruption on Solventogenesis in S. marcescens MG1}

S. marcescens MG1 produced a small amount of ethanol, and the maximal records for WT, $\Delta s w r I$, and $\Delta s w r I$ complemented with exogenous $\mathrm{C}_{6}$-HSL were approximately $1.2,3$, and $1.3 \mathrm{~g} / 1$, respectively. $\Delta s w r I$ produced more ethanol content than WT (Fig. 4A). The QS regulatory system involved in playing a positive role in acetoin synthesis has been well documented [18, 23]. Our results collected from acetoin content determination assays are in agreement with those previous findings. $\Delta s w r I$ produced fewer amounts of acetoin compared with WT at the end of fermentation (Fig. 4B). For 2,3-butanediol production, in the fermentation period of $0-40 \mathrm{~h}$, WT produced more 2,3butanediol content than $\Delta s w r I$, which correlates well with previous findings described by Rob et al. [18]. However, at the end of fermentation, all strains tested here showed comparable 2,3-butanediol production (Fig. 4C). According to our data, AHL-QS in S. marcescens MG1 negatively contributes to ethanol production but positively promotes acetoin and 2,3-butanediol generation. In addition, S. marcescens MG1 seems to reuse its neutral products, with the exception of acetoin [25].

\section{Metabolic Flux Analysis}

As shown in Table 2, $\Delta s w r I$ generated more acid products than WT during the fermentation period of $0-8 \mathrm{~h}$. To be more specific, the amounts of lactic acid, acetic acid, and succinic acid generated by $\Delta s w r I$ were increased by up to
$5.1 \%, 18.0 \%$, and $8.0 \%$ compared with that produced by WT, respectively. As the fermentation process progressed, both WT and $\Delta s w r I$ reduced the generation of their acid products but increased the generation of their neutral products. However, the acid products generated by $\Delta s w r I$ accordingly were much more than those produced by WT. Importantly, by the addition of exogenous $\mathrm{C}_{6}$-HSL to the $\Delta$ swrI medium, the fermentation products generated by $\Delta s w r I$ could be restored to the levels produced by WT. The metabolic flux analysis results showed that AHL-QS exerts a role in the switch from acidogenesis to solventogenesis in S. marcescens MG1 during the fermentation process.

\section{Effect of swrI Disruption on Relevant Enzyme Gene Expression in S. marcescens MG1}

In light of the AHL-QS involved in the switch from acidogenesis to solventogenesis, it is intriguing for us to test the hypothesis that AHL-QS might directly exert effects on the expression of relevant enzyme genes, which are responsible for synthesizing the acid and neutral products we determined. It has been demonstrated that exogenous autoinducer turnover was approximately $20 \mathrm{~h}$ when added to the media [26]. Glucose was completely exhausted at approximately $32 \mathrm{~h}$ during the $S$. marcescens MG1 fermentation process (Fig. 2D). Therefore, samples used for gene expression analysis were prepared at fermentation time points of 8,16 , and $24 \mathrm{~h}$. The mRNA expression levels of $l d h$ encoding lactate dehydrogenase in all tested strains exhibited the same pattern at the analyzed fermentation time points, which showed that the mRNA levels of $l d h$ expressed by $\Delta s w r I$ were approximately four times those generated by WT; the overexpressed $l d h$ mRNA levels in $\Delta s w r I$ could be restored by the addition of exogenous $\mathrm{C}_{6}$-HSL (Figs. 5A-5C). It was reported that acs,

Table 2. Metabolic flux analysis for the products generated by WT and $\Delta s w r I$ with or without exogenous $\mathrm{C}_{6}$-HSL.

\begin{tabular}{lcccccccccccc}
\hline S. marcescen MG1 & $\mathrm{WT}^{\mathrm{a}}$ & $\Delta \mathrm{swrl}^{\mathrm{a}}$ & $\begin{array}{c}\Delta \mathrm{swrI+} \\
\mathrm{C}_{6}-\mathrm{HSL}^{\mathrm{a}}\end{array}$ & $\mathrm{WT}^{\mathrm{b}}$ & $\Delta s w r I^{\mathrm{b}}$ & $\begin{array}{c}\Delta s w r I+ \\
\mathrm{C}_{6}-\mathrm{HSL}^{\mathrm{b}}\end{array}$ & $\mathrm{WT}^{\mathrm{c}}$ & $\Delta s w r \mathrm{C}^{\mathrm{c}}$ & $\begin{array}{c}\Delta s w r I+ \\
\mathrm{C}_{6}-\mathrm{HSL}^{\mathrm{c}}\end{array}$ & WT $^{\mathrm{d}}$ & $\Delta^{4} w r I^{\mathrm{d}}$ & $\begin{array}{c}\Delta s w r I+ \\
\mathrm{C}_{6}-\mathrm{HSL}^{\mathrm{d}}\end{array}$ \\
\hline Biomass & 9.82 & 9.38 & 10.45 & 7.19 & 6.37 & 7.23 & 2.35 & 7.00 & 2.34 & 6.47 & 1.58 & 9.88 \\
Lactic acid & 9.25 & 14.32 & 10.52 & 0.13 & 0.36 & 0.11 & 1.48 & 5.44 & 1.53 & -3.54 & -1.91 & -7.84 \\
Acetic acid & 0.00 & 18.03 & 0.00 & 0.00 & -15.24 & 0.00 & 0.00 & -2.70 & 0.00 & 3.83 & -2.26 & 4.45 \\
Succinic acid & 34.02 & 42.06 & 33.76 & -3.11 & -0.04 & -4.79 & -3.96 & 5.04 & -2.95 & -4.04 & 0.18 & -8.80 \\
Citric acid & 1.83 & 0.94 & 1.64 & -2.09 & 0.73 & -1.68 & -0.14 & 0.39 & 0.01 & 9.49 & 2.74 & 11.94 \\
Ethanol & 7.64 & 5.24 & 9.28 & 4.80 & 7.09 & 3.83 & -4.48 & 14.58 & -0.73 & 2.85 & 8.52 & 0.60 \\
Acetoin & 2.63 & 0.68 & 2.47 & -3.21 & 7.09 & -2.67 & 0.37 & 2.30 & 0.64 & 45.96 & 11.27 & 59.72 \\
2,3-butanediol & 34.82 & 9.35 & 31.88 & 96.30 & 93.64 & 97.96 & 104.38 & 67.95 & 99.16 & 38.98 & 79.88 & 30.04 \\
\hline
\end{tabular}

Note: Metabolic flux was calculated based on the assumption that acid and neutral end products were generated by bacteria consuming $100 \mathrm{~g}$ glucose. Superscript letters ${ }^{\mathrm{a}},{ }^{\mathrm{b}}, \mathrm{c}$, and ${ }^{\mathrm{d}}$ represent the fermentation periods of $0-8 \mathrm{~h}, 8-16 \mathrm{~h}, 16-24 \mathrm{~h}$, and 24-32 h, respectively. 

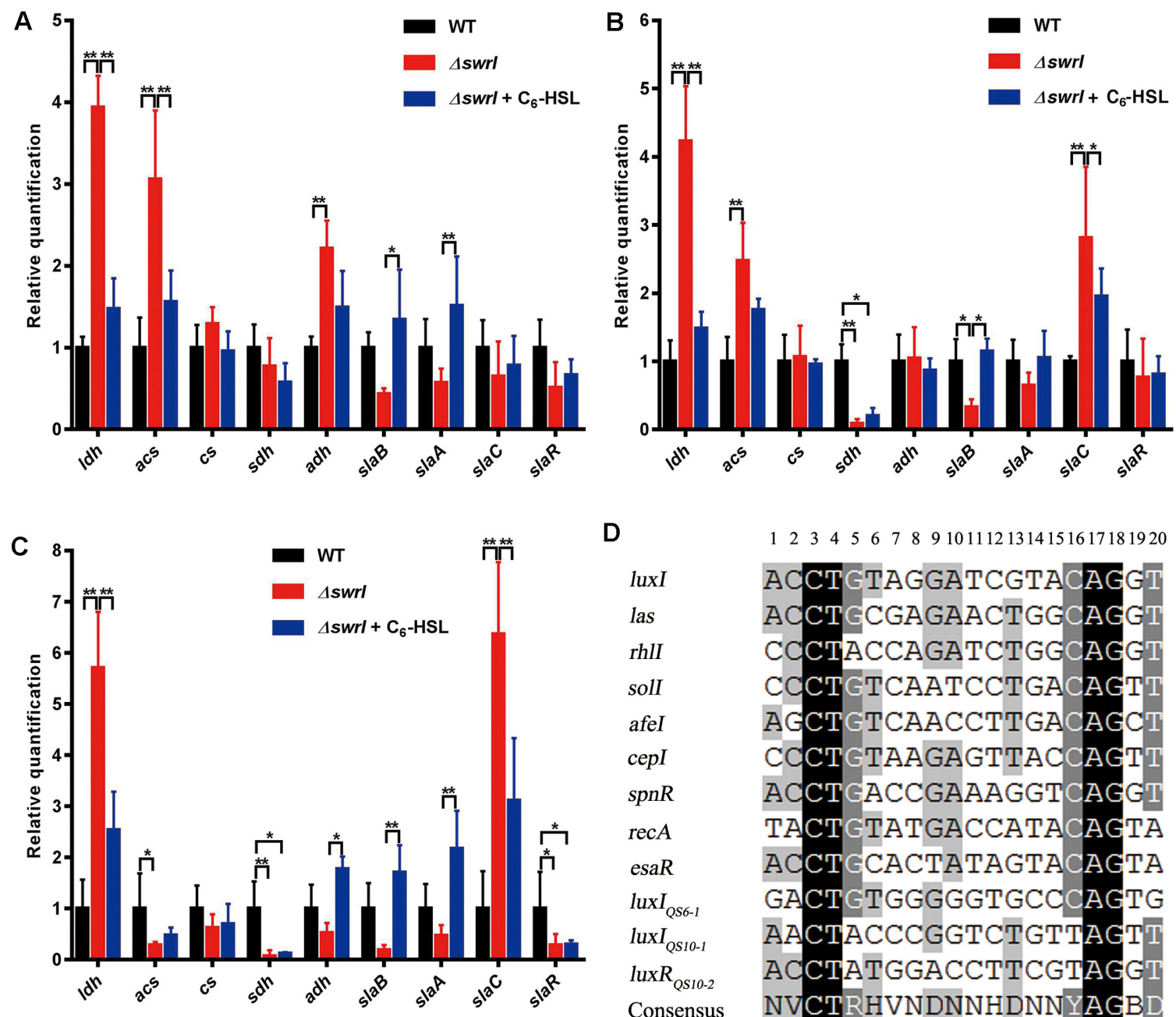

D

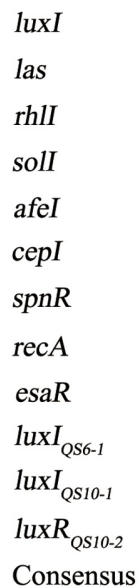

12345677891011121314151617181920

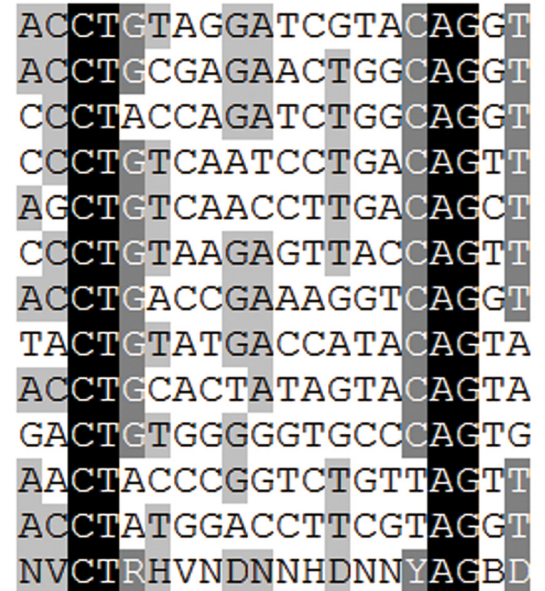

E

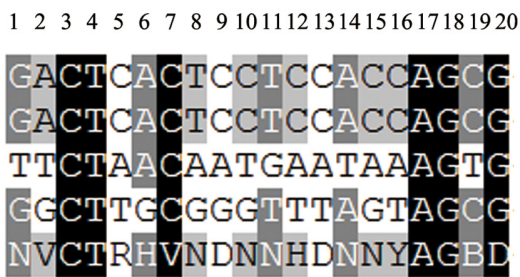

Fig. 5. Effect of swrI disruption on relevant enzyme gene expression in S. marcescens MG1.

Quantitative real-time PCR was used to determine mRNA expression levels of samples collected at fermentation time points of $8 \mathrm{~h}(\mathrm{~A}), 16 \mathrm{~h}(\mathbf{B})$, and $24 \mathrm{~h}(\mathrm{C})$. Relative quantification is reported as fold expression against the WT sample in each set of data. Values are means, and bars indicate standard deviations (SDs) $(n=3)$. Single and double asterisks represent $p<0.05$ and $p<0.01$, respectively. The consensus motif was created by aligning V. fischeri luxI with: Pseudomonas aeruginosa lasI and rhlI; Ralstonia solanacearum solI; Acidithiobacillus ferrooxidans afeI; Burkholderia cepacia cepI; S. marcescens SS-1 spnR and recA; Pantoea stewartii esaR, luxI $Q_{S 6-1}, l u x I_{Q S 10-1}$, and $l u x R_{Q S 10-2}$ as described elsewhere previously [30, 31]. The consensus sequence is shown below the alignment, $\mathrm{N}=\mathrm{A}, \mathrm{T}, \mathrm{C}$ or $\mathrm{G} ; \mathrm{V}=\mathrm{A}, \mathrm{C}$, or $\mathrm{G} ; \mathrm{R}=\mathrm{A}$ or $\mathrm{G} ; \mathrm{H}=\mathrm{A}, \mathrm{T}$, or $\mathrm{C} ; \mathrm{D}=\mathrm{A}, \mathrm{T}$, or $\mathrm{G} ; \mathrm{Y}=\mathrm{C}$ or $\mathrm{T} ; \mathrm{B}=\mathrm{T}, \mathrm{C}$, or $\mathrm{G}$ (D). Four putative luxI box-like motifs existing upstream of S. marcescens MG1 slaR, slaA, acs, and ldh (E).

encoding acetyl-CoA synthase, was under the control of the AinS-QS regulatory system in $V$. fischeri [24]. Our data clearly demonstrate that the AHL-QS regulatory system negatively regulated acs mRNA expression levels. However, AHL-QS seems to be unrelated to regulating the mRNA expression profiles of $a d h, c s$, and $s d h$, which encodes 
alcohol dehydrogenase, citrate synthase, and succinate dehydrogenase, respectively (Figs. 5A-5C). Unexpectedly, $s d h$ mRNA levels were dramatically reduced to baseline levels in $\Delta s w r I$ supplemented with or without exogenous $\mathrm{C}_{6}$-HSL compared to WT. Although the succinic acid contents produced by WT and $\Delta$ swrI supplemented with or without exogenous $\mathrm{C}_{6}$-HSL were comparable during the fermentation time period of $0-32 \mathrm{~h}$ (Fig. 3D), the exact cause for the resulting conflicting results is currently unknown. The genes encoding $\alpha$-acetolactate synthase, $\alpha$-acetolactate decarboxylase, and 2,3-butanediol dehydrogenase in some microorganisms such as Klebsiella terrigena, Enterobacter aerogenes, and Vibrio cholera cluster together to form an operon [27, 28]. However, in S. marcescens MG1, slaA and $s l a B$ were found in one operon apart from slaC, and slaR was divergently transcribed with slaA [14]. QS was found to exert a positive effect on the expression of slaR and sla $A$ $[14,29]$. Our data presented here clearly demonstrate that slaA, slaB, and slaR expression levels were positively influenced by AHL-QS (Figs. 5A-5C). It is of note that AHL-QS seems to exert more profound effects on the slaA and $s l a B$ rather than slaR expression levels, especially at the later fermentation stage (Fig. 5C). This implies that slaR expression might also be controlled by other regulatory circuits. Unexpectedly, slaC expression levels in $\Delta s w r I$, complemented with or without exogenous $\mathrm{C}_{6}-\mathrm{HSL}$, were accordingly higher than in WT (Figs. 5B and 5C), which is in contrast to the conclusion drawn by Rao et al. [14]. The different fermentation media used for assays might account for these conflicting results.

To further uncover how the AHL-QS exerts its effects on the expression of the genes analyzed above, we attempted to determine whether these gene promoter regions contain luxI box-like elements by in silico analysis. We cloned and sequenced the promoter regions (approximately -350 to the translation start site) of the genes we analyzed. All primers used to amplify the target promoter regions are provided in Table S1, and the corresponding sequences are provided in Table S2. Based on the known luxI box-like elements [30, 31 , we aligned these elements and created a consensus motif (Fig. 5D). Four bases are conserved in all elements, including C3, T4, A17, and G18, implying that these four bases might be very important for luxI box-like elements recognized and bound by LuxR-type protein. We scanned target gene promoter regions using the created consensus motif with two criteria: 1) with the best similarity only, and 2) the candidate motif including C3, T4, A17, and G18 bases. Scanning results showed that there are four candidate motifs localized on the slaR, slaA, acs, and $l d h$ promoter regions (Fig. 5E), implying that these four genes are the true targets of AHL-QS in S. marcescens MG1, although it requires further experiments to validate.

Based on our results, we propose a SwrR working model during the $S$. marcescens MG1 fermentation process that, in the absence of autoinducers, SwrR directly binds to the acs, $l d h$, slaR and slaA promoter and activates acs and $l d h$ but represses slaR and slaA. In the presence of autoinducers, SwrR interacts with autoinducers and then detaches from the target gene promoters, resulting in the repression of acs and $l d h$ and removing the repression of slaR and slaA. This model allows $S$. marcescens MG1 to produce more acid products at low cell density, but to generate more neutral products at high cell density. However, this model seems to be a contradiction where SwrR exerts two opposite functions when binding to its different targets. However, it is reminiscent to us of the fact that SpnR represses its target genes but activates its own expression [30] and that Lyslike regulator activates $\alpha$-acetolactate decarboxylase gene expression but represses its own expression [29].

In conclusion, according to the data presented in this study, three conclusions can be drawn. First, AHL-QS is required, but not essential, for $S$. marcescens MG1 growth. Second, AHL-QS facilitates S. marcescens MG1 to produce acid products during the early phase of fermentation and is susceptible to generating more neutral products during the later phase of fermentation. However, the excess acetic acid is removed in an AHL-QS-independent manner. Third, AHL-QS in S. marcescens MG1 exerts its negative effects on regulating the expression of genes responsible for acidogenesis and plays an active role in promoting the expression of genes involved in solventogenesis. By close examination of these analyzed gene promoter regions, slaR, sla $A, a c s$, and $l d h$ promoter regions contain the putative luxI box-like motifs, implying that these four genes are the true targets of AHL-QS in S. marcescens MG1. However, it should be noted that there may be some unknown regulatory circuits involved in the switch from acidogenesis to solventogenesis, other than the AHL-QS regulatory system, in S. marcescens MG1.

\section{Acknowledgments}

This work was supported by the National Natural Science Foundation of China (No. 81673542 and 31870481), New Century Excellent Talents Supporting Plan of the Provincial Education Department of Fujian Province of China (No. 
K8015056A), Key Project of Fujian Province Fund (No. 2019J01070602), and the Science Fund of the Provincial Education Department of Fujian Province of China (No. KLA17041A). This research was also supported by Basic Science Research Program through the National Research Foundation of Korea (NRF) funded by the Ministry of Science, ICT \& Future Planning (2017R1A4A1014806, 2013M3A6A8073184).

\section{Conflict of Interest}

The authors have no financial conflicts of interest to declare.

\section{References}

1. Papenfort K, Bassler BL. 2016. Quorum sensing signalresponse systems in Gram-negative bacteria. Nat. Rev. Microbiol. 14: 576-588.

2. Whiteley M, Diggle SP, Greenberg EP. 2017. Progress in and promise of bacterial quorum sensing research. Nature 551: 313-320.

3. Abisado RG, Benomar S, Klaus JR, Dandekar AA, Chandler JR. 2018. Bacterial quorum sensing and microbial community interactions. MBio 9.

4. Zhou J, Lyu Y, Richlen M, Anderson DM, Cai Z. 2016. Quorum sensing is a language of chemical signals and plays an ecological role in algal-bacterial interactions. CRC Crit. Rev. Plant Sci. 35: 81-105.

5. Mangwani N, Kumari S, Das S. 2016. Bacterial biofilms and quorum sensing: fidelity in bioremediation technology. Biotechnol. Genet. Eng. Rev. 32: 43-73.

6. Givskov M, Olsen L, Molin S. 1988. Cloning and expression in Escherichia coli of the gene for extracellular phospholipase A1 from Serratia liquefaciens. J. Bacteriol. 170: 5855-5862.

7. Rice SA, Koh KS, Queck SY, Labbate M, Lam KW, Kjelleberg S. 2005. Biofilm formation and sloughing in Serratia marcescens are controlled by quorum sensing and nutrient cues. J. Bacteriol. 187: 3477-3485.

8. Givskov M, Eberl L, Molin S. 1997. Control of exoenzyme production, motility and cell differentiation in Serratia liquefaciens. FEMS Microbiol. Lett. 148: 115-122.

9. Lindum PW, Anthoni U, Christophersen C, Eberl L, Molin S, Givskov M. 1998. N-Acyl-L-homoserine lactone autoinducers control production of an extracellular lipopeptide biosurfactant required for swarming motility of Serratia liquefaciens MG1. J. Bacteriol. 180: 6384-6388.

10. Zhang L, Singh R, Sivakumar D, Guo Z, Li J, Chen F, et al. 2017. An artificial synthetic pathway for acetoin, 2,3butanediol, and 2-butanol production from ethanol using cell free multi-enzyme catalysis. Green Chem. 20:230-242.
11. Guo Z, Zhao X, He Y, Yang T, Gao H, Li G, et al. 2017. Efficient (3R)-acetoin production from meso-2,3-butanediol using a new whole-cell biocatalyst with co-expression of meso-2,3-butanediol dehydrogenase, NADH oxidase, and Vitreoscilla hemoglobin. J. Microbiol. Biotechnol. 27: 92-100.

12. Lopez-Contreras AM, Claassen PA, Mooibroek H, De Vos WM. 2000. Utilisation of saccharides in extruded domestic organic waste by Clostridium acetobutylicum ATCC 824 for production of acetone, butanol and ethanol. Appl. Microbiol. Biotechnol. 54: 162-167.

13. Biebl H, Zeng AP, Menzel K, Deckwer WD. 1998. Fermentation of glycerol to 1,3-propanediol and 2,3-butanediol by Klebsiella pneumoniae. Appl. Microbiol. Biotechnol. 50: 24-29.

14. Rao B, Zhang LY, Sun J, Su G, Wei D, Chu J, et al. 2012. Characterization and regulation of the 2,3-butanediol pathway in Serratia marcescens. Appl. Microbiol. Biotechnol. 93: 2147-2159.

15. Celinska E, Grajek W. 2009. Biotechnological production of 2,3-butanediol--current state and prospects. Biotechnol. Adv. 27: 715-725.

16. Stormer FC. 1968. Evidence for induction of the 2,3-butanediolforming enzymes in Aerobacter aerogenes. FEBS Lett. 2: 36-38.

17. Gao S, Guo W, Shi L, Yu Y, Zhang C, Yang H. 2014. Characterization of acetoin production in a budC gene disrupted mutant of Serratia marcescens G12. J. Ind. Microbiol. Biotechnol. 41: 1267-1274.

18. Van Houdt R, Moons P, Hueso Buj M, Michiels CW. 2006. $\mathrm{N}$-acyl-L-homoserine lactone quorum sensing controls butanediol fermentation in Serratia plymuthica RVH1 and Serratia marcescens MG1. J. Bacteriol. 188: 4570-4572.

19. Givskov M, Ostling J, Eberl L, Lindum PW, Christensen AB, Christiansen G, et al. 1998. Two separate regulatory systems participate in control of swarming motility of Serratia liquefaciens MG1. J. Bacteriol. 180: 742-745.

20. Riedel K, Ohnesorg T, Krogfelt KA, Hansen TS, Omori K, Givskov M, et al. 2001. N-acyl-L-homoserine lactonemediated regulation of the lip secretion system in Serratia liquefaciens MG1. J. Bacteriol. 183: 1805-1809.

21. Labbate M, Queck SY, Koh KS, Rice SA, Givskov M, Kjelleberg S. 2004. Quorum sensing-controlled biofilm development in Serratia liquefaciens MG1. J. Bacteriol. 186: 692-698.

22. Zhang L, Shuang C, Xie H, Tian Y, Hu K. 2012. Efficient acetoin production by optimization of medium components and oxygen supply control using a newly isolated Paenibacillus polymyxa CS107. J. Chem. Technol. Biotechnol. 87: 1551-1557.

23. Van Houdt R, Aertsen A, Michiels CW. 2007. Quorumsensing-dependent switch to butanediol fermentation prevents lethal medium acidification in Aeromonas hydrophila AH-1N. Res. Microbiol. 158: 379-385.

24. Studer SV, Mandel MJ, Ruby EG. 2008. AinS quorum sensing regulates the Vibrio fischeri acetate switch. J. Bacteriol. 190: 5915-5923. 
25. Xiao Z, Xu P. 2007. Acetoin metabolism in bacteria. Crit. Rev. Microbiol. 33: 127-140.

26. Byers JT, Lucas C, Salmond GP, Welch M. 2002. Nonenzymatic turnover of an Erwinia carotovora quorumsensing signaling molecule. J. Bacteriol. 184: 1163-1171.

27. Blomqvist $K$, Nikkola $M$, Lehtovaara $P$, Suihko ML, Airaksinen U, Straby KB, et al. 1993. Characterization of the genes of the 2,3-butanediol operons from Klebsiella terrigena and Enterobacter aerogenes. J. Bacteriol. 175: 1392-1404.

28. Kovacikova G, Lin W, Skorupski K. 2005. Dual regulation of genes involved in acetoin biosynthesis and motility/biofilm formation by the virulence activator AphA and the acetateresponsive LysR-type regulator AlsR in Vibrio cholerae. Mol. Microbiol. 57: 420-433.

29. Moons P, Van Houdt R, Vivijs B, Michiels CW, Aertsen A. 2011. Integrated regulation of acetoin fermentation by quorum sensing and $\mathrm{pH}$ in Serratia plymuthica RVH1. Appl. Environ. Microbiol. 77: 3422-3427.

30. Horng YT, Deng SC, Daykin M, Soo PC, Wei JR, Luh KT, et al. 2002. The LuxR family protein SpnR functions as a negative regulator of $\mathrm{N}$-acyl homoserine lactone-dependent quorum sensing in Serratia marcescens. Mol. Microbiol. 45: 1655-1671.

31. Hao Y, Winans SC, Glick BR, Charles TC. 2010. Identification and characterization of new LuxR/LuxI-type quorum sensing systems from metagenomic libraries. Environ. Microbiol. 12: 105-117.

32. Zhang L, Sun J, Hao Y, Zhu J, Chu J, Wei D, et al. 2010. Microbial production of 2,3-butanediol by a surfactant (serrawettin)-deficient mutant of Serratia marcescens H30. J. Ind. Microbiol. Biotechnol. 37: 857-862.

33. Zhang LY, Zhan SR, Chen LZ, Guan X, Hu KH. 2013. Screening of traditional Chinese edible fungi for quorum sensing inhibitors activity. Res. J. Biotechnol. 8: 79-83. 